TRANSACTIONS OF THE

AMERICAN MATHEMATICAL SOCIETY

Volume 362, Number 5, May 2010, Pages 2435-2449

S 0002-9947(09)04842-9

Article electronically published on December 16, 2009

\title{
CONDUCTIVITY INTERFACE PROBLEMS. PART I: SMALL PERTURBATIONS OF AN INTERFACE
}

\author{
HABIB AMMARI, HYEONBAE KANG, MIKYOUNG LIM, AND HABIB ZRIBI
}

\begin{abstract}
We derive high-order terms in the asymptotic expansions of boundary perturbations of steady-state voltage potentials resulting from small perturbations of the shape of a conductivity inclusion with $\mathcal{C}^{2}$-boundary. Our derivation is rigorous and based on layer potential techniques. The asymptotic expansion in this paper is valid for $\mathcal{C}^{1}$-perturbations and inclusions with extreme conductivities. It extends those already derived for small volume conductivity inclusions and leads us to very effective algorithms for determining lower-order Fourier coefficients of the shape perturbation of the inclusion based on boundary measurements. We perform some numerical experiments using the algorithm to test its effectiveness.
\end{abstract}

\section{INTRODUCTION}

The main objective of this paper is to present a schematic way, based on layer potential techniques, of deriving high-order terms in the asymptotic expansions of boundary perturbations of steady-state voltage potentials resulting from small perturbations of the shape of a conductivity inclusion. We then use this formula to design algorithms to recover certain properties of the perturbation of the shape.

More precisely, consider a homogeneous conducting object occupying a bounded domain $\Omega \subset \mathbb{R}^{2}$, with a connected $\mathcal{C}^{2}$-boundary $\partial \Omega$. We assume, for the sake of simplicity, that its conductivity is equal to 1 . Let $D$ with $\mathcal{C}^{2}$-boundary be a conductivity inclusion inside $\Omega$ of conductivity equal to some positive constant $k \neq 1$. We assume that $\operatorname{dist}(D, \partial \Omega) \geq C>0$. The voltage potential in the presence of the inclusion $D$ is denoted by $u$. It is the solution to

$$
\left\{\begin{array}{l}
\nabla \cdot\left(1+(k-1) \chi_{D}\right) \nabla u=0 \quad \text { in } \Omega \\
\left.\frac{\partial u}{\partial \nu}\right|_{\partial \Omega}=g, \int_{\partial \Omega} u=0
\end{array}\right.
$$

where $\chi_{D}$ is the indicator function of $D$. Here $\nu$ denotes the unit outward normal to the domain $\Omega$ and $g$ represents the applied boundary current; it belongs to the set $L_{0}^{2}(\partial \Omega)=\left\{f \in L^{2}(\partial \Omega), \int_{\partial \Omega} f=0\right\}$.

Let $D_{\epsilon}$ be an $\epsilon$-perturbation of $D$, i.e., there is $h \in \mathcal{C}^{1}(\partial D)$ such that $\partial D_{\epsilon}$ is given by

$$
\partial D_{\epsilon}=\{\tilde{x}: \tilde{x}=x+\epsilon h(x) \nu(x), x \in \partial D\}
$$

Received by the editors January 13, 2006 and, in revised form, January 27, 2008.

2000 Mathematics Subject Classification. Primary 35B30.

Key words and phrases. Small perturbations, interface problem, full asymptotic expansions, boundary integral method. 
Let $u_{\epsilon}$ be the solution to

$$
\left\{\begin{array}{l}
\nabla \cdot\left(1+(k-1) \chi_{D_{\epsilon}}\right) \nabla u_{\epsilon}=0 \quad \text { in } \Omega \\
\left.\frac{\partial u_{\epsilon}}{\partial \nu}\right|_{\partial \Omega}=g, \int_{\partial \Omega} u_{\epsilon}=0 .
\end{array}\right.
$$

The main achievement of this paper is a rigorous derivation, based on layer potential techniques, of high-order terms in the asymptotic expansion of $\left.\left(u_{\epsilon}-u\right)\right|_{\partial \Omega}$ as $\epsilon \rightarrow 0$.

The solution $u_{\epsilon}$ to (1.3) can be represented using integral operators (see formula (3.1)), and hence the derivation of an asymptotic formula for $u_{\epsilon}$ is reduced to that of the integral operator $\mathcal{K}_{D_{\epsilon}}^{*}$ defined by

$$
\mathcal{K}_{D_{\epsilon}}^{*}[\tilde{\varphi}](\tilde{x})=\frac{1}{2 \pi} \text { p.v. } \int_{\partial D_{\epsilon}} \frac{\langle\tilde{x}-\tilde{y}, \tilde{\nu}(\tilde{x})\rangle}{|\tilde{x}-\tilde{y}|^{2}} \tilde{\varphi}(\tilde{y}) d \sigma_{\epsilon}(\tilde{y})
$$

where p.v. stands for the Cauchy principal value. The operator $\mathcal{K}_{D_{\epsilon}}^{*}$ is a singular integral operator and known to be bounded on $L^{2}\left(\partial D_{\epsilon}\right)$ [8, 7]. It was proved in [14, 9] that $\mathcal{K}_{D_{\epsilon}}^{*}$ converges to the operator $\mathcal{K}_{D}^{*}$ on the non-perturbed domain $D$, which is defined for a density $\varphi \in L^{2}(\partial D)$ by

$$
\mathcal{K}_{D}^{*}[\varphi](x)=\frac{1}{2 \pi} \int_{\partial D} \frac{\langle x-y, \nu(x)\rangle}{|x-y|^{2}} \varphi(y) d \sigma(y) .
$$

Note that we dropped the p.v. in the above; this is because $\partial D$ is $\mathcal{C}^{2}$. In this paper we will derive a complete asymptotic expansion of the singular integral operator $\mathcal{K}_{D_{\epsilon}}^{*}$ on $L^{2}\left(\partial D_{\epsilon}\right)$ in terms of $\epsilon$. This asymptotic expansion yields an expansion of $u_{\epsilon}-u$ which extends those already derived for small volume inclusions [1, 2, 3, 4, 5, 10, 16, 19].

The asymptotic formula derived in the paper is of significant interest from an "imaging point of view". For instance, if one has detailed knowledge of the "boundary signatures" of conductivity inclusions, then it becomes possible to design effective algorithms to identify certain properties of their shapes. Since the expansion carries precise information on the shape of the inclusion, we will show how it can be efficiently exploited for designing significantly better algorithms. In connection with the reconstruction of interfaces, we refer readers to [6, 12, 17.

This paper is organized as follows: In section 2 we derive higher-order terms in the asymptotic expansion of a certain boundary integral operator which appears in the representation of the steady-state voltage potential. We then use this formula to derive higher-order terms in the asymptotic expansion of the steady-state voltage potential in section 3 . We propose an algorithm to determine the shape perturbation in section 4 . Section 5 presents results of numerical experiments to demonstrate the effectiveness of the algorithm.

\section{High-ORDER TERMS in the EXPANSION OF $\mathcal{K}_{D_{\epsilon}}^{*}$}

Let $a, b \in \mathbb{R}$ with $a<b$, and let $X(t):[a, b] \rightarrow \mathbb{R}^{2}$ be the arclength parametrization of $\partial D$, that is, $X$ is a $\mathcal{C}^{2}$-function satisfying $\left|X^{\prime}(t)\right|=1$ for all $t \in[a, b]$ and such that

$$
\partial D:=\{x=X(t), t \in[a, b]\} .
$$


Then the outward unit normal to $\partial D, \nu(x)$, is given by $\nu(x)=R_{-\frac{\pi}{2}} X^{\prime}(t)$, where $R_{-\frac{\pi}{2}}$ is rotation by $-\pi / 2, X^{\prime}(t)=T(x)$ is the tangential vector at $x$ and $X^{\prime}(t) \perp$ $X^{\prime \prime}(t)$. Let the curvature $\tau(x)$ be defined by

$$
X^{\prime \prime}(t)=\tau(x) \nu(x)
$$

We will sometimes write $h(t)$ for $h(X(t))$ and $h^{\prime}(t)$ for the tangential derivative of $h(x)$.

Then, $\tilde{X}(t)=X(t)+\epsilon h(t) \nu(x)=X(t)+\epsilon h(t) R_{-\frac{\pi}{2}} X^{\prime}(t)$ is a parametrization of $\partial D_{\epsilon}$. By $\tilde{\nu}(\tilde{x})$ we denote the outward unit normal to $\partial D_{\epsilon}$ at $\tilde{x}$. Then we have

$$
\begin{aligned}
\tilde{\nu}(\tilde{x}) & =\frac{R_{-\frac{\pi}{2}} \tilde{X}^{\prime}(t)}{\left|\tilde{X}^{\prime}(t)\right|} \\
& =\frac{(1-\epsilon h(t) \tau(x)) \nu(x)-\epsilon h^{\prime}(t) X^{\prime}(t)}{\sqrt{\epsilon^{2} h^{\prime}(t)^{2}+(1-\epsilon h(t) \tau(x))^{2}}} \\
& =\frac{(1-\epsilon h(t) \tau(x)) \nu(x)-\epsilon h^{\prime}(t) T(x)}{\sqrt{\epsilon^{2} h^{\prime}(t)^{2}+(1-\epsilon h(t) \tau(x))^{2}}},
\end{aligned}
$$

and hence $\tilde{\nu}(\tilde{x})$ can be expanded uniformly as

$$
\tilde{\nu}(\tilde{x})=\sum_{n=0}^{\infty} \epsilon^{n} \nu^{(n)}(x), \quad x \in \partial D,
$$

where the vector-valued functions $\nu^{(n)}$ are uniformly bounded independently of $n$. In particular, the first two terms are given by

$$
\nu^{(0)}(x)=\nu(x), \quad \nu^{(1)}(x)=-h^{\prime}(t) T(x) .
$$

Likewise, we get a uniformly convergent expansion for the length element $d \sigma_{\epsilon}(\tilde{y})$ :

$$
d \sigma_{\epsilon}(\tilde{y})=\left|\tilde{X}^{\prime}(s)\right| d s=\sqrt{(1-\epsilon \tau(s) h(s))^{2}+\epsilon^{2} h^{\prime 2}(s)} d s=\sum_{n=0}^{\infty} \epsilon^{n} \sigma^{(n)}(y) d \sigma(y),
$$

where $\sigma^{(n)}$ are functions bounded independently of $n$ and

$$
\sigma^{(0)}(y)=1, \quad \sigma^{(1)}(y)=-\tau(y) h(y) .
$$

Set

$$
\begin{aligned}
& x=X(t), \tilde{x}=\tilde{X}(t)=x+\epsilon h(t) R_{-\frac{\pi}{2}} X^{\prime}(t), \\
& y=X(s), \tilde{y}=\tilde{X}(s)=y+\epsilon h(s) R_{-\frac{\pi}{2}} X^{\prime}(s) .
\end{aligned}
$$

Since

$$
\tilde{x}-\tilde{y}=x-y+\epsilon(h(t) \nu(x)-h(s) \nu(y)),
$$

we get

$$
|\tilde{x}-\tilde{y}|^{2}=|x-y|^{2}+2 \epsilon\langle x-y, h(t) \nu(x)-h(s) \nu(y)\rangle+\epsilon^{2}|h(t) \nu(x)-h(s) \nu(y)|^{2}
$$

and hence

$$
\frac{1}{|\tilde{x}-\tilde{y}|^{2}}=\frac{1}{|x-y|^{2}} \frac{1}{1+2 \epsilon F(x, y)+\epsilon^{2} G(x, y)},
$$


where

and

$$
F(x, y)=\frac{\langle x-y, h(t) \nu(x)-h(s) \nu(y)\rangle}{|x-y|^{2}}
$$

One can easily see that

$$
G(x, y)=\frac{|h(t) \nu(x)-h(s) \nu(y)|^{2}}{|x-y|^{2}} .
$$

$$
|F(x, y)|+|G(x, y)|^{\frac{1}{2}} \leq C\|X\|_{\mathcal{C}^{2}}\|h\|_{\mathcal{C}^{1}} \quad \text { for all } x, y \in \partial D .
$$

It follows from (2.1), (2.3), (2.5), and (2.6) that

$$
\begin{aligned}
& \frac{\langle\tilde{x}-\tilde{y}, \tilde{\nu}(\tilde{x})\rangle}{|\tilde{x}-\tilde{y}|^{2}} d \sigma_{\epsilon}(\tilde{y})=\left(\frac{\langle x-y, \nu(x)\rangle}{|x-y|^{2}}+\epsilon\left[\frac{\langle h(t) \nu(x)-h(s) \nu(y), \nu(x)\rangle}{|x-y|^{2}}\right.\right. \\
& \left.-\frac{\left\langle x-y, \tau(x) h(t) \nu(x)+h^{\prime}(t) T(x)\right\rangle}{|x-y|^{2}}\right] \\
& \quad-\epsilon^{2} \frac{\left\langle h(t) \nu(x)-h(s) \nu(y), \tau(x) h(t) \nu(x)+h^{\prime}(t) T(x)\right\rangle}{|x-y|^{2}} \\
& \quad \times \frac{1}{1+2 \epsilon F(x, y)+\epsilon^{2} G(x, y)} \frac{\sqrt{(1-\epsilon \tau(y) h(s))^{2}+\epsilon^{2} h^{\prime 2}(s)}}{\sqrt{(1-\epsilon \tau(x) h(t))^{2}+\epsilon^{2} h^{\prime 2}(t)}} d \sigma(y) \\
& :=\left(K_{0}(x, y)+\epsilon K_{1}(x, y)+\epsilon^{2} K_{2}(x, y)\right) \\
& \quad \times \frac{1}{1+2 \epsilon F(x, y)+\epsilon^{2} G(x, y)} \frac{\sqrt{(1-\epsilon \tau(y) h(s))^{2}+\epsilon^{2} h^{\prime 2}(s)}}{\sqrt{(1-\epsilon \tau(x) h(t))^{2}+\epsilon^{2} h^{\prime 2}(t)}} d \sigma(y) .
\end{aligned}
$$

Let

$$
\frac{1}{1+2 \epsilon F(x, y)+\epsilon^{2} G(x, y)} \frac{\sqrt{(1-\epsilon \tau(y) h(s))^{2}+\epsilon^{2} h^{\prime 2}(s)}}{\sqrt{(1-\epsilon \tau(x) h(t))^{2}+\epsilon^{2} h^{\prime 2}(t)}}=\sum_{n=0}^{\infty} \epsilon^{n} F_{n}(x, y)
$$

where the series converges absolutely and uniformly. In particular, we can easily see that

$$
F_{0}(x, y)=1, \quad F_{1}(x, y)=-2 F(x, y)+\tau(x) h(x)-\tau(y) h(y) .
$$

So we now have

$$
\begin{aligned}
& \frac{\langle\tilde{x}-\tilde{y}, \tilde{\nu}(\tilde{x})\rangle}{|\tilde{x}-\tilde{y}|^{2}} d \sigma_{\epsilon}(\tilde{y})=\frac{\langle x-y, \nu(x)\rangle}{|x-y|^{2}} d \sigma(y)+\epsilon\left(K_{0}(x, y) F_{1}(x, y)+K_{1}(x, y)\right) d \sigma(y) \\
& \quad+\epsilon^{2} \sum_{n=0}^{\infty} \epsilon^{n}\left(F_{n+2}(x, y) K_{0}(x, y)+F_{n+1}(x, y) K_{1}(x, y)+F_{n}(x, y) K_{2}(x, y)\right) d \sigma(y) .
\end{aligned}
$$

Therefore, we obtain that

$$
\frac{\langle\tilde{x}-\tilde{y}, \tilde{\nu}(\tilde{x})\rangle}{|\tilde{x}-\tilde{y}|^{2}} d \sigma_{\epsilon}(\tilde{y})=\sum_{n=0}^{\infty} \epsilon^{n} \mathbb{k}_{n}(x, y) d \sigma(y),
$$

where

$$
\mathbb{k}_{0}(x, y)=\frac{\langle x-y, \nu(x)\rangle}{|x-y|^{2}}, \quad \mathbb{k}_{1}(x, y)=K_{0}(x, y) F_{1}(x, y)+K_{1}(x, y),
$$

and for any $n \geq 2$,

$$
\mathbb{k}_{n}(x, y)=F_{n}(x, y) K_{0}(x, y)+F_{n-1}(x, y) K_{1}(x, y)+F_{n-2}(x, y) K_{2}(x, y) .
$$


Introduce a sequence of integral operators $\left(\mathcal{K}_{D}^{(n)}\right)_{n \in \mathbb{N}}$, defined for any $\phi \in L^{2}(\partial D)$ by

$$
\mathcal{K}_{D}^{(n)} \phi(x)=\int_{\partial D} \mathbb{k}_{n}(x, y) \phi(y) d \sigma(y) \quad \text { for } n \geq 0 .
$$

Note that $\mathcal{K}_{D}^{(0)}=\mathcal{K}_{D}^{*}$. Observe that the same operator with the kernel $\mathbb{k}_{n}(x, y)$ replaced with $K_{j}(x, y), j=0,1,2$, is bounded on $L^{2}(\partial D)$. In fact, this is an immediate consequence of the celebrated theorem of Coifman-McIntosh-Meyer [7. Therefore each $\mathcal{K}_{D}^{(n)}$ is bounded on $L^{2}(\partial D)$.

Let $\Psi_{\epsilon}$ be the diffeomorphism from $\partial D$ onto $\partial D_{\epsilon}$ given by $\Psi_{\epsilon}(x)=x+\epsilon h(t) \nu(x)$, where $x=X(t)$. The following theorem holds.

Theorem 2.1. Let $N \in \mathbb{N}$. There exists $C$ depending only on $N,\|X\|_{\mathcal{C}^{2}}$, and $\|h\|_{\mathcal{C}^{1}}$ such that for any $\tilde{\phi} \in L^{2}\left(\partial D_{\epsilon}\right)$,

$$
\left\|\mathcal{K}_{D_{\epsilon}}^{*}[\tilde{\phi}] \circ \Psi_{\epsilon}-\mathcal{K}_{D}^{*}[\phi]-\sum_{n=1}^{N} \epsilon^{n} \mathcal{K}_{D}^{(n)}[\phi]\right\|_{L^{2}(\partial D)} \leq C \epsilon^{N+1}\|\phi\|_{L^{2}(\partial D)},
$$

where $\phi:=\tilde{\phi} \circ \Psi_{\epsilon}$.

\section{Derivation of the full asymptotic formula \\ FOR THE STEADY-STATE VOLTAGE POTENTIALS}

In this section we derive high-order terms in the asymptotic expansion of $\left(u_{\epsilon}-\right.$ $u)\left.\right|_{\partial \Omega}$ as $\epsilon \rightarrow 0$.

Suppose that the conductivity of $D$ is $k$. Let $\lambda:=\frac{k+1}{2(k-1)}$. Define the background voltage potential, $U$, to be the unique solution to

$$
\left\{\begin{array}{l}
\Delta U=0 \quad \text { in } \Omega \\
\left.\frac{\partial U}{\partial \nu}\right|_{\partial \Omega}=g, \int_{\partial \Omega} U=0 .
\end{array}\right.
$$

Let $N(x, y)$ be the Neumann function for $\Delta$ in $\Omega$ corresponding to a Dirac mass at $y$, that is, $N$ is the solution to

$$
\left\{\begin{array}{l}
\Delta_{x} N(x, y)=-\delta_{y} \quad \text { in } \Omega \\
\left.\frac{\partial N}{\partial \nu}\right|_{\partial \Omega}=-\frac{1}{|\partial \Omega|} \\
\int_{\partial \Omega} N(x, y) d \sigma(x)=0 \quad \text { for } y \in \Omega .
\end{array}\right.
$$

Let $\mathcal{N}_{D}$ be defined by

$$
\mathcal{N}_{D}[\varphi](x):=\int_{\partial D} N(x, y) \varphi(y) d \sigma(y), \quad x \in \partial \Omega,
$$

for $\varphi \in L_{0}^{2}(\partial D)$.

Let $u_{\epsilon}$ be the solution to (1.3). Then the following representation formula holds [1]:

$$
u_{\epsilon}(x)=U(x)-\mathcal{N}_{D_{\epsilon}}\left[\tilde{\phi}_{\epsilon}\right](x), \quad x \in \partial \Omega,
$$

where $\tilde{\phi}_{\epsilon} \in L_{0}^{2}\left(\partial D_{\epsilon}\right)$ is given by

$$
H_{\epsilon}(x):=\mathcal{D}_{\Omega}\left[\left.u_{\epsilon}\right|_{\partial \Omega}\right](x)-\mathcal{S}_{\Omega}[g](x), \quad x \in \Omega,
$$


and

$$
\left(\lambda I-\mathcal{K}_{D_{\epsilon}}^{*}\right)\left[\tilde{\phi}_{\epsilon}\right]=\frac{\partial H_{\epsilon}}{\partial \tilde{\nu}} \quad \text { on } \partial D_{\epsilon} .
$$

Here and throughout this paper $\mathcal{S}_{\Omega}$ and $\mathcal{D}_{\Omega}$ denote the single and double layer potentials on $\partial \Omega$ :

$$
\begin{aligned}
& \mathcal{D}_{\Omega}[\varphi](x)=\frac{1}{2 \pi} \int_{\partial \Omega} \frac{\langle y-x, \nu(y)\rangle}{|x-y|^{2}} \varphi(y) d \sigma(y), \quad x \in \mathbb{R}^{2} \backslash \partial \Omega, \\
& \mathcal{S}_{\Omega}[\varphi](x)=\frac{1}{2 \pi} \int_{\partial \Omega} \log |x-y| \varphi(y) d \sigma(y), \quad x \in \mathbb{R}^{2} .
\end{aligned}
$$

Likewise the solution $u$ to (1.1) has the representation

$$
u(x)=U(x)-\mathcal{N}_{D}[\phi](x), \quad x \in \partial \Omega,
$$

where $\phi \in L_{0}^{2}(\partial D)$ is given by

$$
H(x):=\mathcal{D}_{\Omega}\left[\left.u\right|_{\partial \Omega}\right](x)-\mathcal{S}_{\Omega}[g](x), \quad x \in \Omega,
$$

and

$$
\left(\lambda I-\mathcal{K}_{D}^{*}\right)[\phi]=\frac{\partial H}{\partial \nu} \quad \text { on } \partial D
$$

We then get

$$
u_{\epsilon}(x)-u(x)=-\mathcal{N}_{D_{\epsilon}}\left[\tilde{\phi}_{\epsilon}\right](x)+\mathcal{N}_{D}[\phi](x), \quad x \in \partial \Omega .
$$

We now investigate the asymptotic behavior of $\mathcal{N}_{D_{\epsilon}}\left[\tilde{\phi}_{\epsilon}\right]$ as $\epsilon \rightarrow 0$. After the change of variables $\tilde{y}=\Psi_{\epsilon}(y)$, we get from (2.3) and the Taylor expansion of $N(x, \tilde{y})$ in $y \in \partial D$ for each fixed $x \in \partial \Omega$ that

$\mathcal{N}_{D_{\epsilon}}\left[\tilde{\phi}_{\epsilon}\right](x)=\int_{\partial D}\left(\sum_{n=0}^{\infty} \sum_{|\alpha|=n} \frac{\epsilon^{n}}{\alpha !}(h(y) \nu(y))^{\alpha} \partial_{y}^{\alpha} N(x, y)\right) \phi_{\epsilon}(y)\left(\sum_{n=0}^{\infty} \epsilon^{n} \sigma^{(n)}(y)\right) d \sigma(y)$, where $\phi_{\epsilon}=\tilde{\phi}_{\epsilon} \circ \Psi_{\epsilon}$.

One can see from Theorem 2.1 that, for each integer $N, \phi_{\epsilon}$ satisfies

$$
\left(\lambda I-\mathcal{K}_{D}^{*}-\sum_{n=1}^{N} \epsilon^{n} \mathcal{K}_{D}^{(n)}\right)\left[\phi_{\epsilon}\right]+O\left(\epsilon^{N+1}\right)=\left(\nabla H_{\epsilon} \circ \Psi_{\epsilon}\right) \cdot\left(\tilde{\nu} \circ \Psi_{\epsilon}\right) \quad \text { on } \partial D .
$$

We obtain from (2.2) that

$$
\begin{gathered}
\left(\nabla H_{\epsilon} \circ \Psi_{\epsilon}\right)(y) \cdot\left(\tilde{\nu} \circ \Psi_{\epsilon}\right)(y)=\left(\sum_{n=0}^{\infty} \sum_{|\alpha|=n} \frac{\epsilon^{n}}{\alpha !}\left(\nabla \partial^{\alpha} H_{\epsilon}\right)(y)(h(y) \nu(y))^{\alpha}\right) \\
\cdot\left(\sum_{n=0}^{\infty} \epsilon^{n} \nu^{(n)}(y)\right) \\
:=\sum_{n=0}^{\infty} \epsilon^{n} G_{n}(y) .
\end{gathered}
$$

Note that

$$
G_{0}(y)=\frac{\partial H_{\epsilon}}{\partial \nu}(y), \quad G_{1}(y)=h(y)\left\langle D^{2} H_{\epsilon}(y) \nu(y), \nu(y)\right\rangle-h^{\prime}(y)\left\langle\nabla H_{\epsilon}, T(y)\right\rangle,
$$


where $D^{2} H_{\epsilon}$ is the Hessian of $H_{\epsilon}$. Therefore, we obtain the following integral equation to solve:

$$
\left(\lambda I-\mathcal{K}_{D}^{*}-\sum_{n=1}^{N} \epsilon^{n} \mathcal{K}_{D}^{(n)}\right)\left[\phi_{\epsilon}\right]+O\left(\epsilon^{N+1}\right)=\sum_{n=0}^{+\infty} \epsilon^{n} G_{n} \quad \text { on } \partial D .
$$

The equation (3.9) can be solved recursively in the following way: Define

$$
\phi^{(0)}=\left(\lambda I-\mathcal{K}_{D}^{*}\right)^{-1}\left[G_{0}\right]=\left(\lambda I-\mathcal{K}_{D}^{*}\right)^{-1}\left[\left.\frac{\partial H_{\epsilon}}{\partial \nu}\right|_{\partial D}\right]
$$

and, for $1 \leq n \leq N$,

$$
\phi^{(n)}=\left(\lambda I-\mathcal{K}_{D}^{*}\right)^{-1}\left[G_{n}+\sum_{p=0}^{n-1} \mathcal{K}_{D}^{(n-p)} \phi^{(p)}\right] .
$$

We obtain the following lemma.

Lemma 3.1. Let $N \in \mathbb{N}$. There exists $C$, depending only on $N$, the $\mathcal{C}^{2}$-norm of $X$, and the $\mathcal{C}^{1}$-norm of $h$, such that

$$
\left\|\phi_{\epsilon}-\sum_{n=0}^{N} \epsilon^{n} \phi^{(n)}\right\|_{L^{2}(\partial D)} \leq C \epsilon^{N+1},
$$

where $\phi^{(n)}$ are defined by the recursive relation (3.11).

Define, for $n \in \mathbb{N}$ and $x \in \partial \Omega$,

$$
v_{n}(x):=\sum_{i+j+k=n} \int_{\partial D}\left(\sum_{|\alpha|=i} \frac{1}{\alpha !}(h(y) \nu(y))^{\alpha} \partial_{y}^{\alpha} N(x, y)\right) \sigma^{(j)}(y) \phi^{(k)}(y) d \sigma(y) .
$$

It then follows from (3.8) and (3.12) that

$$
\mathcal{N}_{D_{\epsilon}}\left[\tilde{\phi}_{\epsilon}\right](x)=\mathcal{N}_{D}\left(\lambda I-\mathcal{K}_{D}^{*}\right)^{-1}\left[\left.\frac{\partial H_{\epsilon}}{\partial \nu}\right|_{\partial D}\right]+\sum_{n=1}^{N} \epsilon^{n} v_{n}(x)+O\left(\epsilon^{N+1}\right) .
$$

Hence we get from (3.5) that

$$
u_{\epsilon}(x)-u(x)=-\mathcal{N}_{D}\left(\lambda I-\mathcal{K}_{D}^{*}\right)^{-1}\left[\left.\frac{\partial H_{\epsilon}}{\partial \nu}\right|_{\partial D}-\left.\frac{\partial H}{\partial \nu}\right|_{\partial D}\right]-\sum_{n=1}^{N} \epsilon^{n} v_{n}(x)+O\left(\epsilon^{N+1}\right), \quad x \in \partial \Omega .
$$

Observe from (3.2) and (3.3) that

$$
H_{\epsilon}(x)-H(x)=\mathcal{D}_{\Omega}\left[\left.u_{\epsilon}\right|_{\partial \Omega}-\left.u\right|_{\partial \Omega}\right](x), \quad x \in \Omega .
$$

If we define the operator $\mathcal{E}$ on $L_{0}^{2}(\partial \Omega)$ by

$$
\mathcal{E}[v](x):=\mathcal{N}_{D}\left(\lambda I-\mathcal{K}_{D}^{*}\right)^{-1}\left[\left.\frac{\partial}{\partial \nu}\left(\mathcal{D}_{\Omega} v\right)\right|_{\partial D}\right](x), \quad x \in \partial \Omega,
$$

then it follows that

$$
(I+\mathcal{E})\left[u_{\epsilon}-u\right](x)=-\sum_{n=1}^{N} \epsilon^{n} v_{n}(x)+O\left(\epsilon^{N+1}\right), \quad x \in \partial \Omega .
$$

We need the following lemma.

Lemma 3.2. The operator $I+\mathcal{E}$ is invertible on $L_{0}^{2}(\partial \Omega)$. 
Let us continue deriving the asymptotic expansion of $\left.\left(u_{\epsilon}-u\right)\right|_{\partial \Omega}$ and leave the proof of Lemma 3.2 to the end of this section.

We get from (3.14) that

$$
u_{\epsilon}(x)-u(x)=-\sum_{n=1}^{N} \epsilon^{n}(I+\mathcal{E})^{-1}\left[v_{n}\right](x)+O\left(\epsilon^{N+1}\right), \quad x \in \partial \Omega .
$$

Observe that the function $v_{n}$ still depends on $\epsilon$, since $G_{n}$ in (3.7) is defined by $H_{\epsilon}$ and hence $\phi^{(n)}$ depends on $\epsilon$. We can remove this dependence on $\epsilon$ from the asymptotic formula in an iterative way.

Observe from (3.15) that

$$
\left.\left(u_{\epsilon}-u\right)\right|_{\partial \Omega}=O(\epsilon)
$$

and hence, by (3.13),

$$
H_{\epsilon}(x)-H(x)=O(\epsilon) .
$$

Thus if we define $G_{n}^{1}, n \in \mathbb{N}$, by (3.7) with $H_{\epsilon}$ replaced with $H$, and define $\phi_{1}^{(n)}$ and $v_{n}^{1}$ by (3.10), (3.11), and (3.12), then $v_{n}-v_{n}^{1}=O(\epsilon)$. Therefore we get

$$
u_{\epsilon}(x)-u(x)=-\epsilon^{1}(I+\mathcal{E})^{-1}\left[v_{1}^{1}\right](x)+O\left(\epsilon^{2}\right), \quad x \in \partial \Omega .
$$

Repeat the same procedure with $H-\epsilon \mathcal{D}_{\Omega}(I+\mathcal{E})^{-1}\left[v_{n}^{1}\right]$ instead of $H$ to get $v_{n}^{2}$. Then $v_{n}-v_{n}^{2}=O\left(\epsilon^{2}\right)$ and hence

$$
u_{\epsilon}(x)-u(x)=-\sum_{n=1}^{2} \epsilon^{n}(I+\mathcal{E})^{-1}\left[v_{n}^{2}\right](x)+O\left(\epsilon^{3}\right), \quad x \in \partial \Omega .
$$

Repeating the same procedure until we get $v_{n}^{N}$, we obtain the following theorem.

Theorem 3.3. Let $v_{n}^{N}, n=1, \ldots, N$, be the functions obtained by the above procedure. Then the following formula holds uniformly for $x \in \partial \Omega$ :

$$
u_{\epsilon}(x)-u(x)=-\sum_{n=1}^{N} \epsilon^{n}(I+\mathcal{E})^{-1}\left[v_{n}^{N}\right](x)+O\left(\epsilon^{N+1}\right) .
$$

The remainder $O\left(\epsilon^{N+1}\right)$ depends only on $N, \Omega$, the $\mathcal{C}^{2}$-norm of $X$, the $\mathcal{C}^{1}$-norm of $h$, and $\operatorname{dist}(D, \partial \Omega)$.

Let us compute the first-order approximation of $\left.\left(u_{\epsilon}-u\right)\right|_{\partial \Omega}$ explicitly. Note that $\phi_{1}^{(0)}=\phi$, where $\phi$ is defined by (3.4), and

$$
\phi_{1}^{(1)}=\left(\lambda I-\mathcal{K}_{D}^{*}\right)^{-1}\left[h\left\langle\left(D^{2} H\right) \nu, \nu\right\rangle-h^{\prime}\langle\nabla H, T\rangle+\mathcal{K}_{D}^{(1)} \phi\right] .
$$

Therefore, by (2.4) and (3.12), $v_{1}^{1}$ takes the form

$$
\begin{aligned}
v_{1}^{1}(x)= & \int_{\partial D} \nabla_{y} N(x, y) \cdot \nu(y) h(y) \phi(y) d \sigma(y) \\
& -\int_{\partial D} N(x, y) \tau(y) h(y) \phi(y) d \sigma(y)+\int_{\partial D} N(x, y) \phi_{1}^{(1)}(y) d \sigma(y) .
\end{aligned}
$$

Using this formula and (3.16) we find the first-order term in the asymptotic expansion of $u_{\epsilon}-u$ on $\partial \Omega$.

The first term in the asymptotic expansion is exactly the domain derivative of the solution derived in [11, Theorem 1]. To see this, it suffices to prove that

$$
(I+\mathcal{E})[w]=-\frac{1}{k-1} v_{1}^{1} \quad \text { on } \partial \Omega
$$


where $w$ is the solution of

$$
\left\{\begin{array}{l}
\Delta w=0 \quad \text { in }(\Omega \backslash \bar{D}) \cup D \\
\left.w\right|_{+}-\left.w\right|_{-}=-\left.h \frac{\partial u}{\partial \nu}\right|_{-} \text {on } \partial D, \\
\left.\frac{\partial w}{\partial \nu}\right|_{+}-\left.k \frac{\partial w}{\partial \nu}\right|_{-}=-\frac{\partial}{\partial T}\left(h \frac{\partial u}{\partial T}\right) \quad \text { on } \partial D \\
\frac{\partial w}{\partial \nu}=0 \text { on } \partial \Omega .
\end{array}\right.
$$

It is easy to see that

$$
w=\mathcal{D}_{\Omega}\left[\left.w\right|_{\partial \Omega}\right]+\mathcal{D}_{D}\left[\left.h \frac{\partial u}{\partial \nu}\right|_{-}\right]+\mathcal{S}_{D}[\theta], \quad x \in \Omega,
$$

where the density $\theta$ on $\partial D$ is given by

$$
\theta=\left(\lambda I-\mathcal{K}_{D}^{*}\right)^{-1}\left[-\frac{1}{k-1}\left(\frac{\partial}{\partial T} h \frac{\partial u}{\partial T}\right)+\left.\frac{\partial}{\partial \nu}\left(\mathcal{D}_{\Omega}[w]\right)\right|_{\partial D}+\left.\frac{\partial}{\partial \nu}\left(\mathcal{D}_{D}\left[\left.h \frac{\partial u}{\partial \nu}\right|_{-}\right]\right)\right|_{\partial D}\right] .
$$

Thus, for $x \in \partial \Omega$,

$$
\begin{aligned}
w(x) & +\mathcal{N}_{D}\left(\lambda I-\mathcal{K}_{D}^{*}\right)^{-1}\left[\left.\frac{\partial}{\partial \nu}\left(\mathcal{D}_{\Omega}[w]\right)\right|_{\partial D}\right](x)=-\left.\int_{\partial D} \frac{\partial N}{\partial \nu(y)}(x, y) h(y) \frac{\partial u}{\partial \nu}\right|_{-}(y) d \sigma(y) \\
& +\frac{1}{k-1} \int_{\partial D} N(x, y)\left(\lambda I-\mathcal{K}_{D}^{*}\right)^{-1}\left[\frac{\partial}{\partial T} h \frac{\partial u}{\partial T}\right](y) d \sigma(y) \\
& -\int_{\partial D} N(x, y)\left(\lambda I-\mathcal{K}_{D}^{*}\right)^{-1}\left[\left.\frac{\partial}{\partial \nu}\left(\mathcal{D}_{D}\left(\left.h \frac{\partial u}{\partial \nu}\right|_{-}\right)\right]\right|_{\partial D}\right) d \sigma(y) .
\end{aligned}
$$

Since

$$
\left.\frac{\partial u}{\partial \nu}\right|_{-}=\frac{1}{k-1} \phi
$$

and

$$
\frac{\partial}{\partial T} h \frac{\partial u}{\partial T}=h\left(-\left\langle\left(D^{2} H\right) \nu, \nu\right\rangle-\tau \frac{\partial H}{\partial \nu}\right)+h^{\prime} \frac{\partial H}{\partial T}+\frac{\partial}{\partial T} h \frac{\partial}{\partial T} \mathcal{S}_{D}[\phi],
$$

where $\phi$ is defined by (3.4), it is not difficult to see that (3.17) holds by using the expression for $\mathcal{K}_{D}^{(1)}$.

Proof of Lemma 3.2. Since $\mathcal{E}$ is a compact operator, we can apply the Fredholm alternative.

Suppose that $(I+\mathcal{E})[v]=0$. Then, first of all, $v$ is smooth on $\partial \Omega$. Since $\left(-\frac{1}{2} I+\mathcal{K}_{\Omega}\right) \mathcal{N}_{D}=\mathcal{S}_{D}$ on $L_{0}^{2}(\partial D)$, as was proved in [1, 2, 3], we get

$$
\left(-\frac{1}{2} I+\mathcal{K}_{\Omega}\right)[v]+\mathcal{S}_{D}\left(\lambda I-\mathcal{K}_{D}^{*}\right)^{-1}\left[\left.\frac{\partial}{\partial \nu}\left(\mathcal{D}_{\Omega}[v]\right)\right|_{\partial D}\right]=0 \quad \text { on } \partial \Omega .
$$

Since

$$
\left.\mathcal{D}_{\Omega}[f]\right|_{-}(x)=\left(\frac{1}{2} I+\mathcal{K}_{\Omega}\right)[f](x), \quad x \in \partial \Omega,
$$

where the subscript - denotes the limit from the inside of $\Omega$ [18, we get

$$
v(x)=\left.\mathcal{D}_{\Omega}[v]\right|_{-}(x)+\mathcal{S}_{D}\left(\lambda I-\mathcal{K}_{D}^{*}\right)^{-1}\left[\left.\left(\frac{\partial}{\partial \nu} \mathcal{D}_{\Omega}[v]\right)\right|_{\partial D}\right](x), \quad x \in \partial \Omega .
$$


Thus $v$ can be extended to the whole of $\Omega$ to satisfy

$$
v(x)=\mathcal{D}_{\Omega}[v](x)+\mathcal{S}_{D}\left(\lambda I-\mathcal{K}_{D}^{*}\right)^{-1}\left[\left.\left(\frac{\partial}{\partial \nu} \mathcal{D}_{\Omega}[v]\right)\right|_{\partial D}\right](x), \quad x \in \Omega
$$

Let the space $W^{1,2}(\Omega)$ be the set of functions $f \in L^{2}(\Omega)$ such that $\nabla f \in L^{2}(\Omega)$. We now recall the following facts from [13, 15]: The $W^{1,2}$-solution $u$ to (1.1) has the representation

$$
u(x)=H(x)+\mathcal{S}_{D}[\phi](x), \quad x \in \Omega,
$$

where the harmonic function $H \in W^{1,2}(\Omega)$ and $\phi \in L_{0}^{2}(\partial D)$ are given by (3.3) and (3.4). Moreover the harmonic function $H$ and $\phi$ are unique.

Observe that $v$ given in (3.19) takes exactly the form in (3.20) with $H=\mathcal{D}_{\Omega}[v]$. By (3.3) and uniqueness of $H$, we have $\mathcal{S}_{\Omega}\left[\left.\frac{\partial v}{\partial \nu}\right|_{\partial \Omega}\right]=0$ in $\Omega$. It then follows that $\left.\frac{\partial v}{\partial \nu}\right|_{\partial \Omega}=0$ on $\partial \Omega$, and hence $v$ is constant in $\Omega$. Since $v \in L_{0}^{2}(\partial \Omega)$, we get $v=0$. So $I+\mathcal{E}$ is injective and hence invertible. This completes the proof.

\section{Reconstruction of the interface Deformation}

We finally give a reconstruction formula for the shape deformation $h$ from measurements of boundary perturbations $u_{\epsilon}-u$ on $\partial \Omega$. Recall from the previous section that

$$
\left(u_{\epsilon}-u\right)(x)=\epsilon(k-1) w(x)+O\left(\epsilon^{2}\right), \quad x \in \partial \Omega .
$$

For $f \in L_{0}^{2}(\partial \Omega)$, let $v$ be the solution to

$$
\left\{\begin{array}{l}
\nabla \cdot\left(1+(k-1) \chi_{D}\right) \nabla v=0 \quad \text { in } \Omega \\
\left.\frac{\partial v}{\partial \nu}\right|_{\partial \Omega}=f, \int_{\partial \Omega} v=0
\end{array}\right.
$$

In view of (3.18), multiplying (4.1) by $f$ and integrating over $\partial \Omega$ yields

$$
\int_{\partial \Omega} f\left(u_{\epsilon}-u\right) d \sigma=\epsilon(1-k) \int_{\partial D} h\left[\frac{\partial v}{\partial T} \frac{\partial u}{\partial T}+\left.\left.k \frac{\partial v}{\partial \nu}\right|_{-} \frac{\partial u}{\partial \nu}\right|_{-}\right] d \sigma+O\left(\epsilon^{2}\right),
$$

where $u$ is the solution to (1.1). Formula (4.2) can be used in order to reconstruct an approximation of $h$ by choosing $v$ appropriately. Higher-order terms given in Theorem 3.3 can be used to reconstruct a better approximation of $h$.

To illustrate this, let us consider $D$ to be the disk centered at the origin with radius $\rho$. For an integer $n$, set

$$
u_{n}(r, \theta)= \begin{cases}c_{n} \rho^{-|n|} r^{|n|} e^{i n \theta}, & r<\rho, \\ \frac{c_{n}}{2}\left(\rho^{-|n|}(k+1) r^{|n|}-\rho^{|n|}(k-1) r^{-|n|}\right) e^{i n \theta}, & r \geq \rho,\end{cases}
$$

where

$$
c_{n}=\frac{2}{|n|} \frac{1}{\rho^{-|n|}(k+1)+\rho^{|n|}(k-1)} .
$$

One can easily see that $u_{n}$ satisfies $\nabla \cdot\left(1+(k-1) \chi_{D}\right) \nabla u_{n}=0$. We then take $u=u_{n}$ and $v=u_{m}$ in formula (4.2) with $f(\theta)=\left.\frac{\partial u_{m}}{\partial \nu}\right|_{\partial \Omega}$. It then follows from (4.2) that

$$
\int_{\partial \Omega} f\left(u_{\epsilon}-u\right) d \sigma=\epsilon c_{n, m}(k) \int_{\partial D} h e^{i(n+m) \theta} d \theta+O\left(\epsilon^{2}\right),
$$


where

$$
c_{n, m}(k)=\rho^{-1} c_{n} c_{m}(1-k)(k|n||m|-n m) .
$$

Formula (4.4) shows that the Fourier coefficients $h_{p}$ of $h$ can be determined from measurements of $u_{\epsilon}-u$ on $\partial \Omega$ by varying the test function $v=u_{m}$, provided that the order of magnitude of $\left|h_{p}\right|$ is much larger than $\epsilon$. To reconstruct higher Fourier coefficients of $h$ or more accurately the first ones, the high-order asymptotic expansions derived in this paper should be used. The idea is quite simple. We first use the leading-order term to determine the first Fourier coefficients of $h$, say $h_{p}$, for $|p| \leq L$ with a certain $L$. Since $h$ is $\mathcal{C}^{1},\left|h_{p}\right| \leq \frac{C}{|p|}$ for some constant $C$, hence we may choose $L$ so that $L=O(1 / \epsilon)$. Then, we plug these coefficients into the second-order term by approximating $h$ by $\sum_{|p| \leq L} h_{p} e^{i p \theta}$, and use the second-order asymptotic expansion in Theorem 3.3 to both determine further Fourier coefficients and get more accurate approximations of $\left(h_{p}\right)_{|p| \leq L}$. This procedure can be repeated recursively.

\section{Numerical Results}

We now present results of numerical implementation of the interface reconstruction method described in the previous section. In this section we fix the conductivity of the inclusion to be $k=4$, and the background domain $\Omega$ is assumed to be the ellipse given by

$$
\frac{(x+0.3)^{2}}{a^{2}}+\frac{y^{2}}{b^{2}} \leq 1, \quad a=1.2, b=1 .
$$

In view of (4.4), the Fourier coefficients $h_{p}$ of $h$ can be approximately reconstructed from the boundary measurements by using the formula

$$
h_{p}=\frac{1}{2 \pi \epsilon c_{n, m}(k)} \int_{\partial \Omega} f\left(u_{\epsilon}-u\right) d \sigma,
$$

where

$$
(n, m)= \begin{cases}(-1,1), & \text { for } p=0 \\ (-1,-p+1), & \text { for } p \leq-1 \\ (1,-p-1), & \text { for } p \geq 1\end{cases}
$$

In order to obtain the boundary data $\left.u_{\epsilon}\right|_{\partial \Omega}$, we solve the forward problem (1.3) using the boundary integral representation of $u_{\epsilon}$. Using the jump formula for single and double layer potentials, $u_{\epsilon}$ can be represented as

$$
u(x)=\mathcal{S}_{D}[\varphi](x)+\mathcal{S}_{\Omega}[\psi](x)+C, \quad x \in \Omega,
$$

where $(\varphi, \psi) \in L_{0}^{2}\left(\partial D_{\epsilon}\right) \times L_{0}^{2}(\partial \Omega)$ is the solution to the integral equation

$$
\left\{\begin{array}{l}
\left(\frac{k+1}{2(k-1)} I-\mathcal{K}_{D}^{*}\right)[\varphi]-\left.\frac{\partial}{\partial \nu} \mathcal{S}_{\Omega}[\psi]\right|_{\partial D}=0 \quad \text { on } \partial D, \\
\left.\frac{\partial}{\partial \nu} \mathcal{S}_{D}[\varphi]\right|_{\partial \Omega}+\left(-\frac{1}{2} I+\mathcal{K}_{\Omega}^{*}\right)[\psi]=g \text { on } \partial \Omega,
\end{array}\right.
$$

and $C$ is a constant chosen to make $\int_{\partial \Omega} u=0$. We discretize (5.2) and solve the resulting linear system to get $(\varphi, \psi)$. Then, the boundary measurement $\left.u_{\epsilon}\right|_{\partial \Omega}$ is calculated from (5.1).

Example 1. In this example the interface perturbation is given by $h(\theta)=1+$ $2 \cos (j \theta), j=0,3,6,9$, and $\epsilon=0.03,0.05$. The perturbation function $h$ is approximated by $\sum_{|p| \leq 6} h_{p} e^{i p \theta}$ for $j=0,3,6$ and $\sum_{|p| \leq 9} h_{p} e^{i p \theta}$ for $j=9$. Figure 1 

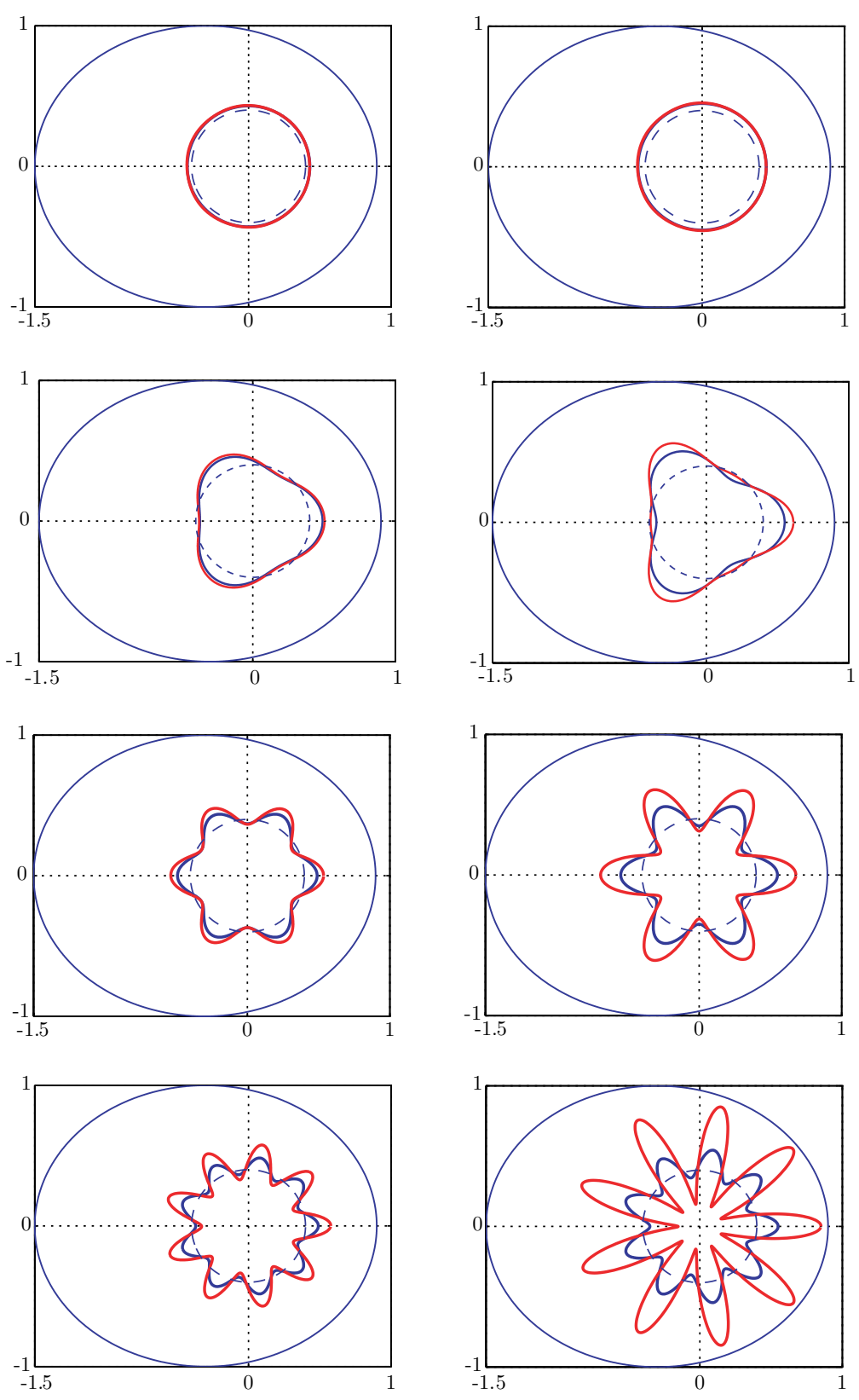

Figure 1. The solid blue (black) curves represent the inclusions, which are perturbations of disks, the dashed (blue) curves. The red (grey) curves are the reconstructed inclusions. The perturbation is given by $\epsilon h$, where $\epsilon=0.03$ in the left column and $\epsilon=0.05$ in the right column.

clearly exhibits that the reconstruction method of this paper works pretty well for perturbations with fewer oscillations when the magnitude $\epsilon$ of the perturbation is 
small. The performance of the method becomes worse as the oscillation gets larger and the magnitude of the perturbation gets bigger.
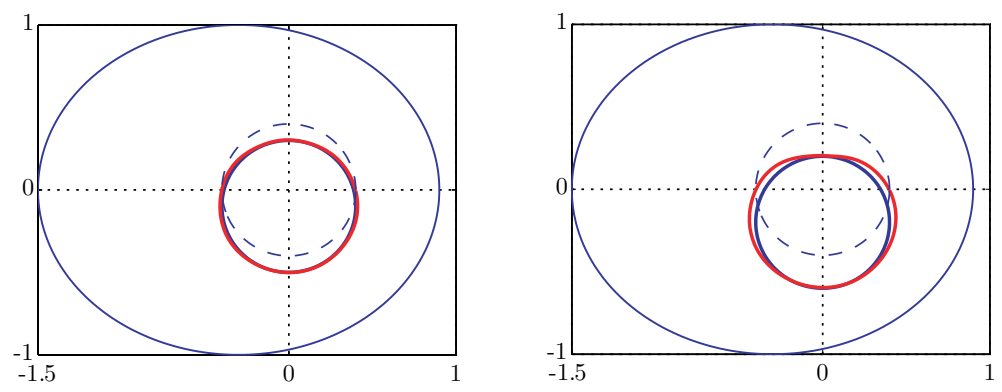

FiguRE 2. Reconstruction result for when the inclusion is shifted by 0.1 (left diagram) and 0.2 (right diagram).

Example 2. This example shows the reconstruction results for shifted inclusions. The initial circular inclusion $D$ is shifted by 0.1 and by 0.2 . The perturbation function $h$ is approximated by $\sum_{|p| \leq 6} h_{p} e^{i p \theta}$. Results are shown in Figure 2 ,
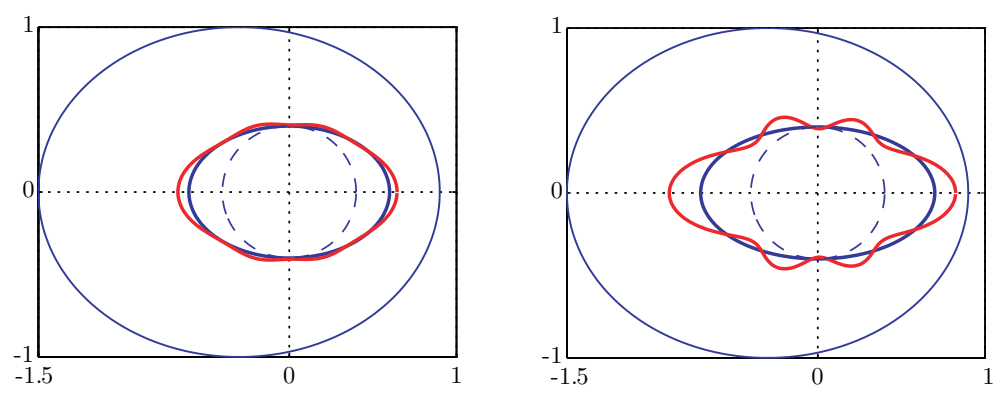

Figure 3. Reconstruction result for when the inclusions are ellipses.

Example 3. This example shows the reconstruction results when the inclusion is an ellipse given by $\frac{x^{2}}{(0.4+a)^{2}}+\frac{y^{2}}{0.4^{2}}=1$ for $a=0.2$ and 0.3 . The perturbation function $h$ is approximated by $\sum_{|p| \leq 6} h_{p} e^{i p \theta}$. The performance of the reconstruction method is much better when $a=0.2$, which means that the reconstruction method of this paper is sensitive to the magnitude of the perturbation. Results are shown in Figure 3 .

\section{CONCLUSION}

In this paper we have derived high-order terms in the asymptotic expansions of boundary perturbations of steady-state voltage potentials resulting from small perturbations of the shape of a conductivity inclusion, using layer potential techniques. We then used the asymptotic formula to design a method to reconstruct the perturbation of the shape. The results of numerical experiments show that 
the method works pretty well in detecting the low-frequency terms of the Fourier coefficients of the perturbation when the magnitude of the perturbation is small.

\section{ACKNOWLEDGEMENTS}

This research was partially supported by the Korea Science and Engineering Foundation grant R01-2006-000-10002-0, the STAR project 190117RD, and the ANR project EchoScan.

\section{REFERENCES}

[1] H. Ammari and H. Kang, High-order terms in the asymptotic expansions of the steady-state voltage potentials in the presence of conductivity inhomogeneities of small diameter, SIAM J. Math. Anal., 34 (2003), 1152-1166. MR2001663 (2004e:35027)

[2] — Reconstruction of Small Inhomogeneities from Boundary Measurements, Lecture Notes in Mathematics, Vol. 1846, Springer-Verlag, Berlin, 2004. MR2168949 (2006k:35295)

[3] _ Polarization and Moment Tensors with Applications to Inverse Problems and Effective Medium Theory, Applied Mathematical Sciences, Vol. 162, Springer-Verlag, New York, 2007. MR2327884 (2009f:35339)

[4] Y. Capdeboscq and M.S. Vogelius, A general representation formula for the boundary voltage perturbations caused by internal conductivity inhomogeneities of low volume fraction, Math. Modelling Num. Anal., 37 (2003), 159-173. MR1972656 (2004b:35334)

[5] D.J. Cedio-Fengya, S. Moskow, and M. Vogelius, Identification of conductivity imperfections of small diameter by boundary measurements. Continuous dependence and computational reconstruction, Inverse Problems, 14 (1998), 553-595. MR.1629995 (99d:78011)

[6] T. Chan and X.-C. Tai, Level set and total variation regularization for elliptic inverse problems with discontinuous coefficients, J. Comput. Phys., 193 (2003), 40-66. MR2022688 (2004j:65170)

[7] R.R. Coifman, A. McIntosh, and Y. Meyer, L'intégrale de Cauchy définit un opérateur bourné sur $L^{2}$ pour les courbes lipschitziennes, Ann. Math., 116 (1982), 361-387. MR672839 (84m:42027)

[8] E.B. Fabes, M. Jodeit, and N.M. Riviére, Potential techniques for boundary value problems on $\mathcal{C}^{1}$ domains, Acta Math., 141 (1978), 165-186. MR501367 (80b:31006)

[9] E. Fabes, H. Kang, and J.K. Seo, Inverse conductivity problem: error estimates and approximate identification for perturbed disks, SIAM J. Math. Anal., 30 (4) (1999), 699-720. MR1684722 (2000d:86015)

[10] A. Friedman and M. Vogelius, Identification of small inhomogeneities of extreme conductivity by boundary measurements: a theorem on continuous dependence, Arch. Rat. Mech. Anal., 105 (1989), 563-579. MR973245 (90c:35198)

[11] F. Hettlich and W. Rundell, The determination of a discontinuity in a conductivity from a single boundary measurement, Inverse Problems, 14 (1998), 67-82. MR.1607628 (99b:35210)

[12] K. Ito, K. Kunish, and Z. Li, Level-set function approach to an inverse interface problem, Inverse Problems, 17 (2001), 1225-1242. MR1862188 (2002h:35335)

[13] H. Kang and J.K. Seo, Layer potential technique for the inverse conductivity problem, Inverse Problems, 12 (1996), 267-278. MR1391539 (97d:35242)

[14] On stability of a transmission problem, Jour. Korean Math. Soc., 34 (1997), 695-706. MR1466611 (98g:35056)

[15] , Recent progress in the inverse conductivity problem with single measurement, in Inverse Problems and Related Fields, CRC Press, 2000, 69-80. MR.1761339 (2001f:35427)

[16] O. Kwon, J.K. Seo, and J.R. Yoon, A real-time algorithm for the location search of discontinuous conductivities with one measurement, Commun. Pure Appl. Math., LV (2002), 1-29. MR.1857878 (2002g:78026)

[17] C.F. Tolmasky and A. Wiegmann, Recovery of small perturbations of an interface for an elliptic inverse problem via linearization, Inverse Problems, 15 (1999), 465-487. MR 1684468 (2000g:65111) 
[18] G.C. Verchota, Layer potentials and boundary value problems for Laplace's equation in Lipschitz domains, J. Funct. Anal., 59 (1984), 572-611. MR769382 (86e:35038)

[19] M. Vogelius and D. Volkov, Asymptotic formulas for perturbations in the electromagnetic fields due to the presence of inhomogeneities, Math. Model. Numer. Anal., 34 (2000), 723748. MR:1784483 (2001f:78024)

Centre de Mathématiques Appliquées, CNRs UMR 7641 and Ecole Polytechnique, 91128 Palaiseau Cedex, France

E-mail address: ammari@cmapx.polytechnique.fr

Department of Mathematical Sciences and Rim, Seoul National University, Seoul 151-747, KOREA

E-mail address: hkang@math.snu.ac.kr

Current address: Department of Mathematics, Inha University, Incheon 402-751, Korea

E-mail address: hbkang@inha.ac.kr

Department of Mathematics, Colorado State University, Fort Collins, Colorado 80523

E-mail address: lim@math.colostate.edu

Current address: Department of Mathematical Sciences, Korean Advanced Institute of Science and Technology, 335 Gwahangno (373-1 Gueseong-dong), Yuseong-gu, Daejeon 305-701, Korea

E-mail address: mklim@kaist.ac.kr

Centre de Mathématiques Appliquées, CNRS UMR 7641 and Ecole Polytechnique, 91128 Palaiseau Cedex, France

E-mail address: zribi@cmapx.polytechnique.fr

Current address: Department of Mathematical Sciences, Korean Advanced Institute of Science and Technology, 335 Gwahangno (373-1 Gueseong-dong), Yuseong-gu, Daejeon 305-701, Korea 